\title{
Radiation hardening of FBG in harsh environments
}

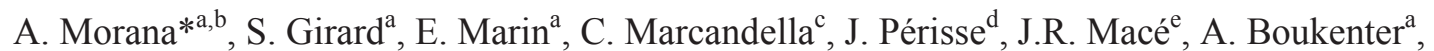 \\ M. Cannas ${ }^{\mathrm{b}}$, Y. Ouerdane ${ }^{\mathrm{a}}$ \\ ${ }^{a}$ Laboratoire Hubert Curien, Université Jean Monnet, CNRS UMR 5516, 18 Rue Prof. B. Lauras, 42000 Saint-Etienne, \\ France; ${ }^{b}$ Dipartimento di Fisica e Chimica, Università di Palermo, via Archirafi 36, 90123 Palermo, Italy; ${ }^{\mathrm{c}} \mathrm{CEA}$, DAM, \\ DIF, F91297 Arpajon, France; ${ }^{\mathrm{d} A r e v a ~ N P, ~} 10$ rue Juliette Récamier 69456 Lyon Cedex 06, France; ${ }^{\mathrm{e} A r e v a, ~ T o u r ~ A r e v a, ~} 1$ \\ place Jean Millier, 92084 Paris La défense Cedex, France
}

\begin{abstract}
The difficulties encountered in the implementation of a temperature or strain sensor based on Fiber Bragg Grating in a harsh radiative environment are introduced. We present the choices made to select both a radiation-resistant fiber in terms of transmission and also the grating inscription conditions necessary to write radiation tolerant FBGs in such fibers with a femto-second laser. The response of different classes of gratings was also studied under radiation at high doses (>1MGy). The comparison between F- and Ge-doped fibers was highlighted.
\end{abstract}

Keywords: fiber, Fiber Bragg Grating, RIA, radiation, silica

\section{INTRODUCTION}

The nuclear industries in the last decades showed an increasing interest in the sensing technology based on optical fibers. Among all the optical fiber sensors (OFSs), the Fiber Bragg Gratings (FBGs) were the most investigated under radiation $^{1}$; indeed, the increase of attenuation induced by ionizing radiation limits the intensity-based OFSs in harsh environments, favoring sensors whose information is encoded in the wavelength measurement. The FBG working is based on the dependence of the Bragg wavelength $\left(\lambda_{B}\right)$ on external parameters, as temperature, strain and pressure: $\lambda_{\mathrm{B}}=2 \mathrm{n}_{\mathrm{eff}} \Lambda$, where $\mathrm{n}_{\mathrm{eff}}$ is the effective index and $\Lambda$ is the grating period. The radiation influences the grating response in different ways. Firstly, it increases the optical fiber transmission losses; consequently, it decreases the signal-to-noise ratio and degrades the grating performance, even if the information is wavelength encoded; in some cases, the losses can be so high to make it impossible to measure the grating peak ${ }^{2}$. To reduce this effect, small pieces of photosensitive fibers, where the gratings can be easily written, are often spliced to more radiation-resistant fibers. Secondly, radiations cause defects and density variations, which lead to changes in the effective index and the grating period, and consequently induce a Bragg wavelength shift (BWS). The behavior of the BWS as a function of the dose is strongly dependent on the fiber composition and the conditions of the grating inscription; except few cases, the Bragg peak redshifts. The recent discovery to write gratings with femto-second IR laser, in place of UV laser light, allows the grating inscription not only in photosensitive fibers, as the Ge-doped ones, but also in the radiation-resistant pure or F-doped silica ones, thus reducing the radiation-induced losses. Since then, very few works ${ }^{3}$ were dedicated to study the radiation sensitivity of these gratings. In spite of all the previous studies, the research is still very active on this field, under the need to find radiation-resistant gratings to be used in harsh environments as strain or temperature sensors and to explain the different behaviors of the radiation-induced peak shifts. In this work, we present experimental results on gratings written in different types of fibers by femto-second radiation at $800 \mathrm{~nm}$, making some comparison with the classical gratings, written with UV light.

\section{EXPERIMENTAL PROCEDURE}

Results on three different types of single-mode fibers will be shown here: the first fiber is characterized by a Ge-doped core, the second one by a F-doping both in the core and in the cladding and the third one has a core in pure silica and a Fdoped cladding (PSC fiber). All the fibers have a $125 \mu \mathrm{m}$ diameter cladding and a diameter core of 8-9 $\mu \mathrm{m}$.

20 meters of the Ge-doped and F-doped fibers were gamma-irradiated with the Brigitte ${ }^{60} \mathrm{Co}$ facility in SCK $\cdot \mathrm{CEN}(\mathrm{Mol}$, Belgium). The accumulated doses varied between 3 and 10 MGy. Spectral attenuation measurements at room temperature (RT) were performed some months after the irradiation, by the "cut-back" method, to characterize the permanent radiation induced damages.

*adriana.morana@univ-st-etienne.fr; phone 0033477915814

23rd International Conference on Optical Fibre Sensors, edited by José Miguel López-Higuera,

Julian Jones, Manuel López-Amo, José Luis Santos, Proc. of SPIE Vol. 9157, 91578I

(C) 2014 SPIE · CCC code: 0277-786X/14/\$18 · doi: 10.1117/12.2059600 
The accumulated doses varied between 3 and 10 MGy. Spectral attenuation measurements at room temperature were performed some months after the irradiation, by the "cut-back" method to characterize the permanent radiation induced damages. The transmission spectra were realized with two white light sources, a spectrometer QE65000 from Ocean Optics for the visible range and an OSA from Yokogawa for the IR range.

The FBGs were written in all the three fiber types by an ultra-fast Ti:sapphire laser emitting 150 fs long pulses at $800 \mathrm{~nm}$ with a repetition rate of $1 \mathrm{kHz}$, through two different phase-masks. To irradiate all the core, the focusing lens can be translate perpendicularly to the direction of light propagation at a frequency ranging between $10 \mathrm{mHz}$ and $10 \mathrm{~Hz}$. To compare the results with a standard technique, only in the Ge-doped fiber that is naturally photosensitive to the UV light, a grating was written by using a frequency doubled CW argon-ion laser (Coherent), emitting at $244 \mathrm{~nm}$ (maximum power of $250 \mathrm{~mW}$ ). Due to the high laser power, the grating was realized directly, without moving the fiber, by using a beam expander. However, to increase the fiber photosensitivity, it was $\mathrm{H}_{2}$-loaded for a week at RT at 130 mbar, before inscription, and annealed at $80^{\circ} \mathrm{C}$ for 7 hours, after the grating inscription, to remove the remaining hydrogen. In contrast to this, the fs-FBGs were not thermally treated after inscription. The transmission spectra of the gratings used in the experiment are shown in figure 1.

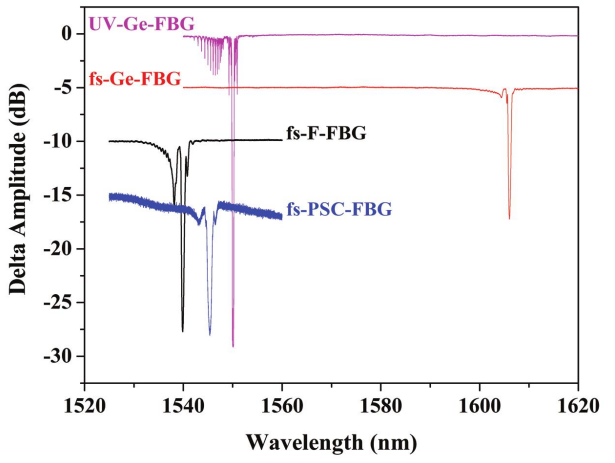

Figure 1. Transmission spectra of the FBGs. To have a clearer graph, their zero amplitude lines were shifted by $5 \mathrm{~dB}$.

To investigate the radiation sensitivity of these gratings, they were irradiated by using a $10 \mathrm{keV}$ X-ray machine Aracor at CEA, Arpajon in France, at RT, about $25^{\circ} \mathrm{C}$, with a dose rate of $50 \mathrm{~Gy}\left(\mathrm{SiO}_{2}\right) / \mathrm{s}$, up to $1 \mathrm{MGy}$. To follow the radiationinduced peak shift during the irradiation, the four gratings were fixed stress-free on the plate of the irradiation system and their transmission spectra were recorded, as shown in figure 2, in parallel by using a tunable laser Tunics Plus from NetTest and the CT400 high performance optical tester from Yenista Optics, with a wavelength resolution of $1 \mathrm{or} 2 \mathrm{pm}$, for the gratings written in the F-doped or Ge-doped fibers, respectively.

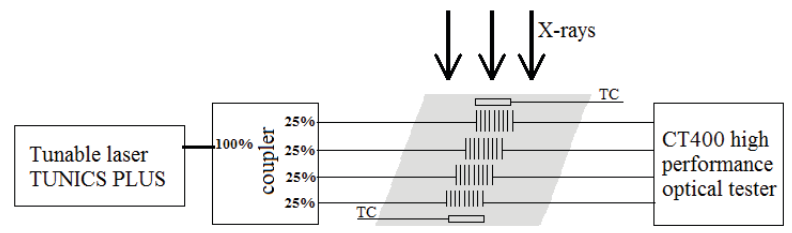

Figure 2. Experimental set-up.

During the irradiation a temperature increase was recorded by two thermocouples placed near the gratings. So, to isolate only the radiation-induced Bragg wavelength shift (BWS), the temperature-induced contribution was calculated and subtracted, once the temperature sensitivity was known. This coefficient was obtained by varying the temperature from $20^{\circ} \mathrm{C}$ to $40^{\circ} \mathrm{C}$ just before the irradiation and by doing linear fit on the BWS as a function of the temperature. The peak position was determined by fitting a $\sim 170 \mathrm{pm}$ range of the transmission spectrum near the main dip with a third order polynomial approximation of the peak, to account for a possible peak asymmetry.

\section{RESULTS AND DISCUSSION}

Figure 3 displays the radiation-induced attenuation recorded with the cut-back technique in the Ge- and F-doped singlemode fibers irradiated at three different $\gamma$-doses, up to $10 \mathrm{MGy}$. Despite the spectra missing part, due to the range of the two detectors, the curves consist of three contributions: an UV-tail which increases with decreasing wavelength, at least an absorption band in the visible range, around $600 \mathrm{~nm}$; an IR-absorption which increases with increasing wavelength. 


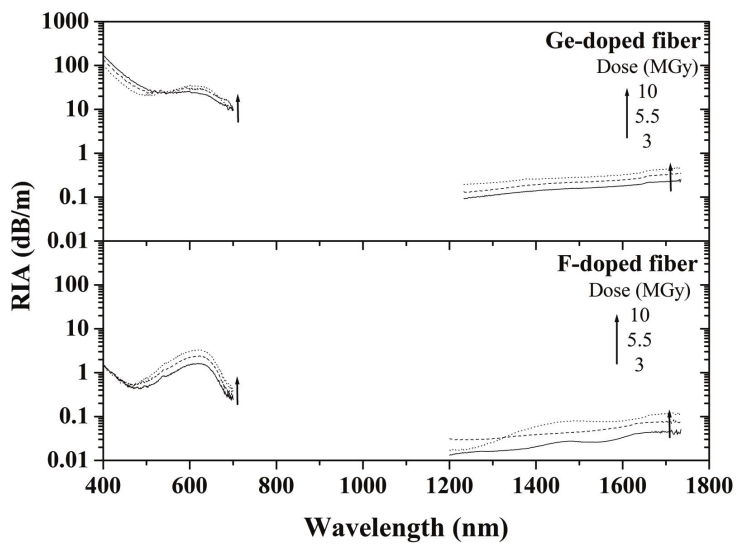

Figure 3. Radiation-induced attenuation, measured by the cut-back technique some months after irradiation at RT, in $\gamma$ irradiated single-mode fibers, doped with (a) germanium and (b) Fluorine, at three doses.

All these contributions increase with increasing irradiation dose, expect the UV tail in the Ge-doped fiber. For the Fdoped fiber, an absorption band around $1400 \mathrm{~nm}$ seems present; in fact this part of the spectrum has to be considered only an upper limit, because of the influence of the fiber bending losses. The gratings used in the present work are active in the range between 1500 and $1650 \mathrm{~nm}$; as a consequence, it is interesting to focus our attention on the RIA in the IR region. Unlike most of the absorption bands in the UV-visible part of the spectrum, the origin of the IR-RIA is not clear yet; only very few studies were concerned with this IR band. Chernov ${ }^{4}$ attributed to self-trapped holes (STHs) a NIRband peaked at a wavelength longer than $1500 \mathrm{~nm}$ but observable only at low temperatures, below $160 \mathrm{~K}$. Even if the STHs are unstable at RT and disappear rapidly after irradiation, Regnier et al. ${ }^{5}$ supposed to associate with these defects a band peaked around $1800 \mathrm{~nm}$ and observed at RT in $\gamma$-irradiated fibers (with a pure or Ge-doped silica core) at a dose of $100 \mathrm{~Gy}$. According to the authors, these defects were observable at RT, thanks to their high measurement sensitivity obtained by using several $\mathrm{km}$ long fibers. In our case, the limited range of the detector lets record only a tail of the IR band, so it is meaningless to fit this curve with a Gaussian function. Moreover, we do not observe the other bands at 660 $\mathrm{nm}$ and $760 \mathrm{~nm}$ associated to the same defects ${ }^{6}$; so we cannot confirm the attribution of the IR absorption to the STHs. Despite of its origin, it is clear that the contribution of the IR absorption is larger in the Ge-doped fiber than in the Fdoped one by a factor of $\sim 4$ around $1550 \mathrm{~nm}(0.30 \mathrm{~dB} / \mathrm{m}$ against $0.08 \mathrm{~dB} / \mathrm{m})$. Hence, the better choice is to use fibers not doped with germanium among the radiation-resistant ones. If the fiber has not a core doped with germanium but in pure or in F-doped silica, it will not be sensitive to the CW UV light, so very powerful laser has to be used for the grating inscription, as the femto-second IR lasers.

Figure 4 shows the behavior of the grating listed above during the irradiation.

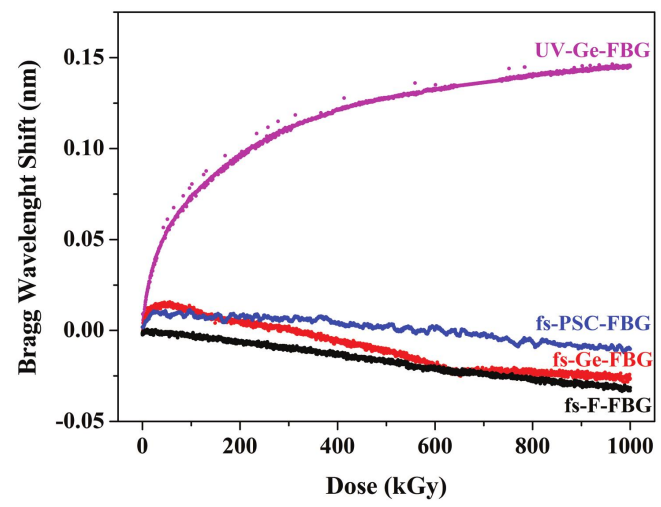

Figure 4. Radiation-induced Bragg wavelength shift as a function of the dose, dose-rate being $50 \mathrm{~Gy} / \mathrm{s}$. 
The results obtained for the FBG written with the UV light in the Ge-doped fiber (UV-Ge-FBG) are consistent with those reported in literature ${ }^{1}$ : the Bragg peak red-shifts with an initial fast increase followed by a slower tendency to saturate. The peak shift should saturate at a dose higher than $1 \mathrm{MGy}$, around a value slightly bigger than $150 \mathrm{pm}$. For the three fs-FBGs, instead, the general effect is a peak blue-shift. However, an initial red-shift is observed for the gratings written in the PSC and Ge-doped fibers up to the dose of about 23 and $56 \mathrm{kGy}$, respectively, at which the inversion of shift direction occurs. The BWS towards the blue does not seem to show a saturating behavior up to a 1 MGy dose; moreover, the slope of the blue shift is larger for the Ge-doped FBG than for the Ge-free FBGs, by a factor $\sim 2(-0.05$ $\mathrm{pm} / \mathrm{kGy}$ against $-0.03 \mathrm{pm} / \mathrm{kGy}$ for the F-doped core fiber and $-0.02 \mathrm{pm} / \mathrm{kGy}$ for the pure silica core fiber). At the accumulated dose of $1 \mathrm{MGy}$, the BWS is about $10 \mathrm{pm}$ for the grating written in the PSC fiber, 24 pm for that in the Gedoped fiber and $32 \mathrm{pm}$ for that in the F-doped fiber. A similar behavior was observed by Henschel et al. on fs-FBGs ${ }^{3}$; however, the BWS obtained at $1 \mathrm{MGy}$ dose, in their case, was lower, between $-10 \mathrm{pm}$ and $+15 \mathrm{pm}$. This difference can be due to the different irradiation conditions: the dose rate used in our experiment was higher, $50 \mathrm{~Gy} / \mathrm{s}$ against their value of $2 \mathrm{~Gy} / \mathrm{s}$, and it has been already demonstrated for the classical UV-FBGs, that higher is dose-rate, bigger is the BWS ${ }^{7}$. Moreover, the gratings studied by Henschel et al. were thermally treated, whereas our fs-FBGs did not withstand any treatments after inscription. Generally, higher is the pre-annealing temperature, higher is the radiation sensitivity of the grating ${ }^{8}$, but by a comparison, it seems that the pre-treatment changes the dose and the BWS value, at which the shift direction occurs.

\section{CONCLUSION}

In the first part of this work we showed the preliminary study that has to be realized to determine in which fiber and how a Fiber Bragg Grating has to be written to create a strain or temperature sensor suitable for a harsh environment. RIA measurement were realized ex situ on two types of fibers and highlighted that in the IR region of the spectrum the RIA is larger in the Ge-doped fiber by a factor about 4 . As a consequence, to reduce the induced losses, a Ge-free fiber has to be chosen, that means that UV lasers cannot be used for the grating inscription. Hence, some gratings were written by fsradiation at $800 \mathrm{~nm}$ in three different types of fibers and the Bragg wavelength shift was studied under irradiation. No big change was recorded for the different fibers, but the behaviour is completely different from that of a classical UVFBG written in the Ge-doped fiber: a blue-shift of the Bragg peak is observed for the fs-FBGs. By a comparison with the previous results reported in literature, we are led to believe that the cause can be the pre-treatment, that is mandatory for the UV-FBG written in $\mathrm{H}_{2}$-loaded fibers, whereas it was not realized on the fs-FBGs. More studies are in progress to find a more radiation-resistant grating, probably by using thermal pre-treatments, and to determine the origin of this blue-shift that can be hardly explained with the defects theory.

\section{REFERENCES}

[1] Gusarov, A. and Hoeffgen, S. K., "Radiation Effects on Fiber Gratings," IEEE Trans. Nucl. Sci. 60 (3), 2037 2053 (2013).

[2] Vasiliev, S. A., Dianov, E. M., Golant, K. M., Medvedkov, O. I., Tomashuk, A. L., Karpov, V. I., Grekov, M. V., Kurkov, A. S., Leconte, B. and Niay, P., "Performance of Bragg and long-period gratings written in N- and Ge-doped silica fibers under $\gamma$-radiation", IEEE Trans. Nucl. Sci. 45 (3), 1580-1583 (1998).

[3] Henschel, H., Grobnic, D., Hoeffgen, S. K., Kuhnhenn, J., Mihailov, S. J. and Weinand, U., "Development of Highly Radiation Resistant Fiber Bragg Gratings," IEEE Trans. Nucl. Sci. 58 (4), 2103-2110 (2011).

[4] Chernov, P. V., "Spectroscopic manifestations of self-trapped holes in silica," Phys. Stat. Sol. B115, 663-675 (1989).

[5] Regnier, E., Flammer, I., Girard, S., Gooijer, F., Achten, F. and Kuyt, G., "Low-dose radiation-induced attenuation at infrared wavelengths for P-doped, Ge-doped and pure silica-core optical fibres", IEEE Trans. Nucl. Sci. 54 (4), 1115-1117 (2007).

[6] Griscom, D. L., " $\gamma$-Ray-induced visible/infrared optical absorption bands in pure and F-doped silica-core fibers: are they due to self-trapped holes?, " J. Non-Cryst. Solids 349, 139-147 (2004).

[7] Fernandez Fernandez, A., Brichard, B., Berghmans, F. and Décreton, M., "Dose-rate dependencies in gammairradiated fiber Bragg grating filters," IEEE Trans. Nucl. Sci. 49 (6), 2874-2877 (2002).

[8] Henschel, H., Hoeffgen, S. K., Krebber, K., Kuhnhenn, J. and Weinand, U., "Influence of Fiber Composition and Grating Fabrication on the Radiation Sensitivity of Fiber Bragg Gratings," IEEE Trans. Nucl. Sci. 55 (4), 2235-2242 (2008). 